\title{
Tumor maligno da bainha de nervo periférico em cães: relato de três casos
}

\author{
[Malignant peripheral nerve sheath tumor in dogs: three cases report] \\ C.M.O. Silva, F.M.C. Caldeira, E.G. Melo, N.M. Ocarino, A.E. Silva, R.M.C. Guedes, \\ G.E. Lavalle, R. Serakides* \\ Escola de Veterinária - UFMG \\ Caixa Postal 567 \\ 30123-970 - Belo Horizonte, MG
}

\begin{abstract}
RESUMO
Descrevem-se os achados anatomoclínicos e imunoistoquímicos dos tumores da bainha de nervos periféricos em três cães atendidos em hospital. Os animais apresentavam sintomatologia variável de acordo com a localização do tumor. À microscopia, as neoplasias eram invasivas e constituídas por células pleomórficas e anaplásicas que variavam de poligonais a fusiformes, arranjadas em padrão sólido difuso, por vezes em paliçada. Foi firmado o diagnóstico de tumor da bainha de nervos periféricos nos três casos.
\end{abstract}

Palavras-chave: cão, tumor da bainha de nervos periféricos, imunoistoquímica

\begin{abstract}
Anatomo-clinical and immunohistochemical findings on three dogs with peripheral nerve sheath tumors assisted at the veterinary hospital are described. Animals showed different clinical signs depending on the location of the tumor. According to microscopy, neoplasia in all the three case were invasive and comprised of pleomorphic, anaplastic with polygonal to very elongated fusiform shapes arranged in a diffuse solid pattern, forming interwoven bundles in some areas. Immunohistochemistry was used to better classify these tumors. The diagnosis of peripheral nerve sheath tumor was confirmed by histological and immunohistochemical findings.
\end{abstract}

Keywords: dog, peripheral nerve sheath tumor, immunohistochemistry

\section{INTRODUÇ̃̃O}

Neoplasias primárias de nervos periféricos são infreqüentes nos animais, embora já tenham sido relatadas em cães, em bovinos (Cordy, 1990; Jubb et al., 1993), em gatos e em cavalo (Garcia et al., 2004). Esses tumores podem originar-se das células de Schwann, recebendo a denominação de schwanomas benigno ou maligno, ou originar-se de fibroblastos do endoneuro ou epineuro, denominados neurofibroma ou neurofibrossarcoma (Lecouteur, 1996; Sawamoto et al., 1999; Simpson et al., 1999). Histologicamente as neoplasias dos nervos periféricos são caracterizadas por extensos fascículos de células alongadas em padrão compactado, em paliçadas; ou células alongadas ou ovais distribuídas de forma mais dispersa em uma matriz edematosa (Sawamoto et al., 1999). Contudo, variações das características histológicas da neoplasia e diferenças entre espécies (Summers et al.,1995) promovem controvérsias quanto à nomenclatura dos tumores da bainha nervosa (Koestner et al., 1999; Kim et al., 2003). Assim, o tumor da bainha do nervo periférico maligno ou benigno é o termo mais utilizado em medicina veterinária (Brehm et al., 1995; Simpson et al., 1999). A distinção entre

Recebido em 30 de março de 2007

Aceito em 31 de maio de 2007

* Autor para correspondência (corresponding author)

E-mail: serakide@dedalus.lcc.ufmg.br 
maligno e benigno é importante porque o prognóstico é bem distinto (Chijiwa et al., 2004). Quando o tumor é maligno o prognóstico tende a ser desfavorável (Patnaik et al., 2002).

Cães das raças de grande porte e com idades entre de 5 e 12 anos são os mais acometidos (Lecouteur, 1996). Os tumores da bainha de nervo periférico geralmente apresentam crescimento lento e localizam-se, preferencialmente, no plexo braquial ou nas raízes dos nervos da região cervical caudal e torácica cranial (Platt et al., 1999). Os sinais clínicos dependem da localização da neoplasia, podendo ocorrer alterações no membro acometido, atrofia muscular, dor à manipulação da região cervical e do membro. Déficits neurológicos também podem ser observados quando a neoplasia comprime ou invade a medula espinhal (Brehm et al., 1995; Platt et al., 1999). O tratamento é restrito à terapia cirúrgica (Lecouteur, 1996), envolvendo a amputação e ressecção do plexo envolvido e a laminectomia ou hemilaminectomia para remoção da raiz do nervo (Platt et al., 1999).

O objetivo deste relato é descrever e discutir os achados anatomoclínicos e imunoistoquímicos dos tumores da bainha de nervos periféricos em três cães.

\section{CASUÍSTICA}

Caso 1

Um cão da raça Pastor-Alemão, macho, de cinco anos de idade, pesando $30 \mathrm{~kg}$, foi encaminhado ao setor de neurologia do hospital veterinário com histórico de claudicação do membro torácico esquerdo que, em aproximadamente sete dias, evoluiu para decúbito lateral. A alimentação, micção e defecação apresentavamse normais. Ao exame clínico, observou-se estado de consciência normal, incapacidade para permanecer em estação, déficit proprioceptivo e atrofia muscular dos membros, principalmente do torácico esquerdo. À avaliação dos reflexos miotáticos, havia hiperreflexia nos membros pélvicos e arreflexia nos torácicos, suspeitandose de lesão na medula espinhal, na região da intumescência cervical. Ao exame radiológico contrastado, havia desvio dorsal e interrupção ventral das linhas no percurso do contraste entre C5 e C6 e presença de área radiopaca de forma ovalada de $1 \mathrm{~cm}$ de diâmetro, sugestivo de alteração intramedular nesta região.

Com base nos achados descritos e na suspeita de neoplasia intradural com prognóstico desfavorável, o animal foi submetido à eutanásia. À necropsia, observou-se na região do plexo braquial esquerdo, um nódulo de aproximadamente $6 \mathrm{~cm}$ de diâmetro, esbranquiçado, com superfície irregular e firme. À secção longitudinal da coluna vertebral, evidenciou-se uma massa neoplásica esbranquiçada com cerca de $3 \mathrm{~cm}$ de extensão, firme, irregular e sem limites precisos, que se infiltrava para o canal medular entre C5 e C6, com compressão da medula espinhal. Não foram observados nódulos em nenhum outro sítio.

Fragmentos das neoplasias foram fixados em formalina a $10 \%$ para processamento pela técnica rotineira de inclusão em parafina e coloração por hematoxilina-eosina e pelo Alcian Blue. Microscopicamente, a proliferação neoplásica localizada predominantemente no espaço epidural, apresentava, em algumas áreas, caráter invasivo onde se observava destruição de parte da substância branca da medula, caracterizada por degeneração axonal e necrose neuronal, confirmando os achados da mielografia. Extensas áreas de necrose associadas a hemorragias multifocais também foram observadas no interior da neoplasia. As células neoplásicas eram pleomórficas, variando de fusiformes a poligonais, sem padrão de organização definido, por vezes formando feixes, dispostas em paliçada ou em ninhos (Fig. 1A). Algumas células eram gigantes e multinucleadas. $O$ citoplasma apresentava-se basofílico, finamente granular com bordas pouco definidas, sendo a razão núcleo-citoplasma elevada. Os núcleos eram grandes, claros, variando de oval a alongado, com nucléolo proeminente e, por vezes, múltiplos com muitos macronucléolos (Fig. 1B). $\mathrm{O}$ índice mitótico era alto, em média 6/campo, com algumas figuras de mitose atípicas (aumento de 40×). Em algumas áreas havia tecido com aspecto mixomatoso rico em mucopolissacarídeos, corados em azul pelo Alcian Blue, foco de metaplasia cartilaginosa e infiltrado inflamatório linfocítico multifocal. Adjacente à neoplasia, havia resquícios de trabéculas ósseas necróticas.

O nódulo da região axilar apresentava proliferação de células neoplásicas com 
características semelhantes às já descritas, permitindo firmar o diagnóstico de tumor maligno da bainha de nervo periférico.

\section{Caso 2}

Cão da raça Poodle de cinco anos de idade, pesando $5,2 \mathrm{~kg}$, com histórico de claudicação, dor e dificuldade de apoio do membro pélvico direito. A alimentação, micção e defecação apresentavam-se normais. Ao exame clínico, foi observado estado geral bom e um aumento de volume firme, de aproximadamente $20 \mathrm{~cm}$ de diâmetro na região da articulação femurotibiopatelar direita. Baseando-se no exame radiológico, que demonstrou destruição óssea intensa, e no diagnóstico citológico de neoplasia mesenquimal maligna, foi realizada a amputação do membro. Fragmentos da neoplasia e do osso, da articulação e dos tecidos moles adjacentes foram fixados em formalina a $10 \%$ para processamento pela técnica rotineira de inclusão em parafina e coloração por hematoxilina-eosina.

À microscopia, a neoplasia infiltrava-se na articulação femurotibiopatelar, no tecido muscular, no canal medular e no tecido ósseo do fêmur e da tíbia, onde havia necrose óssea e osteoclasia intensas. As células neoplásicas encontravam-se arranjadas em padrão sólido, por vezes em paliçada, dispostas ao redor de alguns vasos. Eram pleomórficas e anaplásicas, variando de poligonais a alongadas. $O$ citoplasma era escasso e basofílico, com bordas pouco definidas. A relação núcleo-citoplasma era elevada. Os núcleos eram grandes e pleomórficos, em sua maioria hipocromáticos, com cromatina frouxa, nucléolos proeminentes e múltiplos, variando de um a três. $\mathrm{O}$ índice mitótico era alto, em média 5/campo, com algumas figuras atípicas (aumento de 40×).

\section{Caso 3}

Um cão sem raça definida de 13 anos de idade, pesando $21 \mathrm{~kg}$ foi encaminhado ao hospital veterinário por apresentar um nódulo de crescimento lento, de aproximadamente $10 \mathrm{~cm}$ de diâmetro, friável, localizado na região escapuloumeral esquerda. Após o resultado citológico de neoplasia mesenquimal maligna, foi realizada a exérese cirúrgica do nódulo, com ampla margem de segurança. Macroscopicamente, o tumor apresentava-se de forma arredondada, com aproximadamente $10 \mathrm{~cm}$ de diâmetro que, ao corte, possuia formações císticas com conteúdo líquido amarelado e hemorragias. À microscopia, a neoplasia apresentava limites imprecisos e era constituída por células ovóides ou fusiformes arranjadas em padrão sólido difuso, por vezes em paliçada. As células eram pleomórficas e, em algumas áreas, apresentavam citoplasma claro e vesiculoso.

Nesse caso, assim como no caso 1, foi possível firmar o diagnóstico de tumor maligno da bainha de nervo periférico, baseando-se nos achados anatomopatológicos, a avaliação imunoistoquímica, foi realizada com o objetivo de caracterizar a neoplasia. No caso 2, esses achados não possibilitaram estabelecer o diagnóstico definitivo da neoplasia, recorrendo-se ao exame imunoistoquímico.

A imunoistoquímica foi realizada com o objetivo de definir o diagnóstico e/ou caracterizar as neoplasias, utilizando os marcadores em suas diluições apropriadas - anti-proteína S100 para os três casos relatados, anti-vimentina (V9), coquetel de anti-citoqueratinas (AE1 e AE3), anti-antígeno epitelial de membrana (E29) e antiCD 34 para os casos 2 e 3, anti-MIC 2 para o caso 2 e anti-actina alfa de músculo liso ${ }^{1}$ para os casos 1 e 2 - (Tab. 1). Os anticorpos foram utilizados em secções parafinizadas de $4 \mu \mathrm{m}$ do tecido neoplásico. Quando necessário, empregou-se a técnica de recuperação antigênica pelo método de calor úmido. O complexo

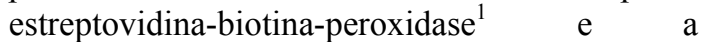
diaminobenzidina $^{2}$ foram utilizados para detecção dos antígenos. Secções foram contracoradas com hematoxilina de Harris.

O sumário dos resultados da imunoistoquímica estão listados na Tab. 1. As células da neoplasia dos casos 1 e 2 apresentaram expressão forte e difusa de proteína S-100. A neoplasia do caso 3 apresentou expressão forte e difusa nos vasos e no citoplasma das células tanto de vimentina quanto de citoqueratinas, e expressão fraca e multifocal de CD34 na célula endotelial.

${ }^{1}$ Dako, Califórnia, EUA

${ }^{2} \mathrm{DAB}$-Lab Vision Corporation, Fremont, CA, EUA 
Tabela 1. Expressão imunoistoquímica das células neoplásicas de casos de tumor maligno da bainha do nervo periférico de cães conforme o anticorpo

\begin{tabular}{llll}
\hline \multirow{2}{*}{ Anticorpo } & \multicolumn{3}{c}{ Expressão imunoistoquímica } \\
\cline { 2 - 4 } & Caso1 & Caso 2 & Caso 3 \\
\hline Anti-proteína S-100 & forte e difusa & forte e difusa & negativo \\
Anti-vimentina & NR & negativo & forte e difusa \\
Anti-citoqueratina AE1/AE3 & NR & negativo & forte e difusa \\
Anti-antígeno epitelial de membrana & NR & negativo & negativo \\
Anti-MIC2 & NR & negativo & NR \\
Anti-CD34 & NR & negativo & fraca e multifocal \\
Anti-alfa actina de músculo liso & negativo & negativo & NR
\end{tabular}

NR: não realizado

\section{DISCUSSÃO}

Os sinais clínicos apresentados pelo animal 1 de claudicação do membro torácico esquerdo com evolução para decúbito e atrofia muscular neurogênica dos membros torácicos são manifestações presentes nos casos de tumores da bainha do nervo periférico (Vasilopulos et al., 2002) do plexo braquial. Segundo Simpson et al. (1999), esse tipo de tumor possui início insidioso, promovendo anormalidades na deambulação no membro torácico unilateral, com atrofia muscular neurogênica e ausência de dor, como também evidenciado neste relato. A evolução do caso, com incapacidade de permanecer em estação e presença de déficit proprioceptivo, são indícios de crescimento progressivo do tumor, com invasão do canal medular. A manifestação neurológica em todos os membros foi determinada pelo acometimento da medula espinhal cervical, que apresentou à histologia infiltração da neoplasia, com destruição de parte da substância branca, caracterizada por degeneração axonal e necrose neuronal (Simpson et al., 1999). As áreas de aspecto mixomatoso e os focos de metaplasia cartilaginosa observadas à histologia do caso 1 já foram descritas por Summers et al. (1995) em uma revisão da morfologia desses tumores relatando diferenciação ostóide, condróide, mixóide e padrão escamoso. Os achados anatomopatológicos foram essenciais para se estabelecer o diagnóstico de tumor da bainha do plexo braquial, pois dificilmente esse tipo de tumor pode ser diagnosticado apenas com base nos achados clínicos (Simpson et al., 1999).

O animal 2 apresentava claudicação e dor ao apoiar o membro devido a neoplasia localizada na articulação femurotibiopatelar que invadia o tecido muscular, o canal medular e o tecido ósseo adjacente. Nesse animal, devido à localização da neoplasia ser na região da articulação fêmuro-tíbio-patelar, incomum para os tumores de nervo periférico (Sawamoto et al., 1999; Simpson et al., 1999), e de acordo com as características radiológicas e citológicas, a principal suspeita clínica foi de uma neoplasia de origem óssea ou articular. Contudo, o diagnóstico de neoplasia maligna foi determinado pelas características histológicas como, elevada atipia celular, alto grau de invasibilidade de tecidos adjacentes e elevado índice mitótico com presença de mitoses atípicas.

Baseando-se na imunoreatividade positiva dos animais 1 e 2 para a proteína S-100 os tumores foram considerados derivados da bainha de nervo periférico (Fig. 1C). Esse marcador é específico para tipo celular de origem neural (Patnaik et al., 2002; Garcia et al., 2004), semelhante ao que ocorre no homem, no qual 50 a $90 \%$ dos tumores malignos de bainha nervosa apresentam positividade para proteína S-100 (Sawamoto et al., 1999). Entretanto, estudo realizado por Stoica et al (2001) demonstrou que imunoistologicamente tumores malignos de bainha nervosa podem ser negativos para proteína $\mathrm{S}-100$ e positivos para vimentina, o que também foi observado no animal do caso 3 descrito neste trabalho (Fig. 1D). A falha na reatividade para proteína S-100 pode ser indicativa de variabilidade de expressão dependendo do grau de diferenciação celular (Garcia et al., 2004). Os tumores malignos anaplásicos ou heterogêneos da bainha do nervo periférico tendem a ser negativos para S-100. Especula-se que a negatividade para essa proteína sustenta o diagnóstico de malignidade (Chijiwa et al., 2004). A origem histológica heterogênea desse tipo de tumor talvez explique a diferenciação divergente, a inconsistente imunorreatividade e os achados ultra-estruturais (Kim et al., 2003). 

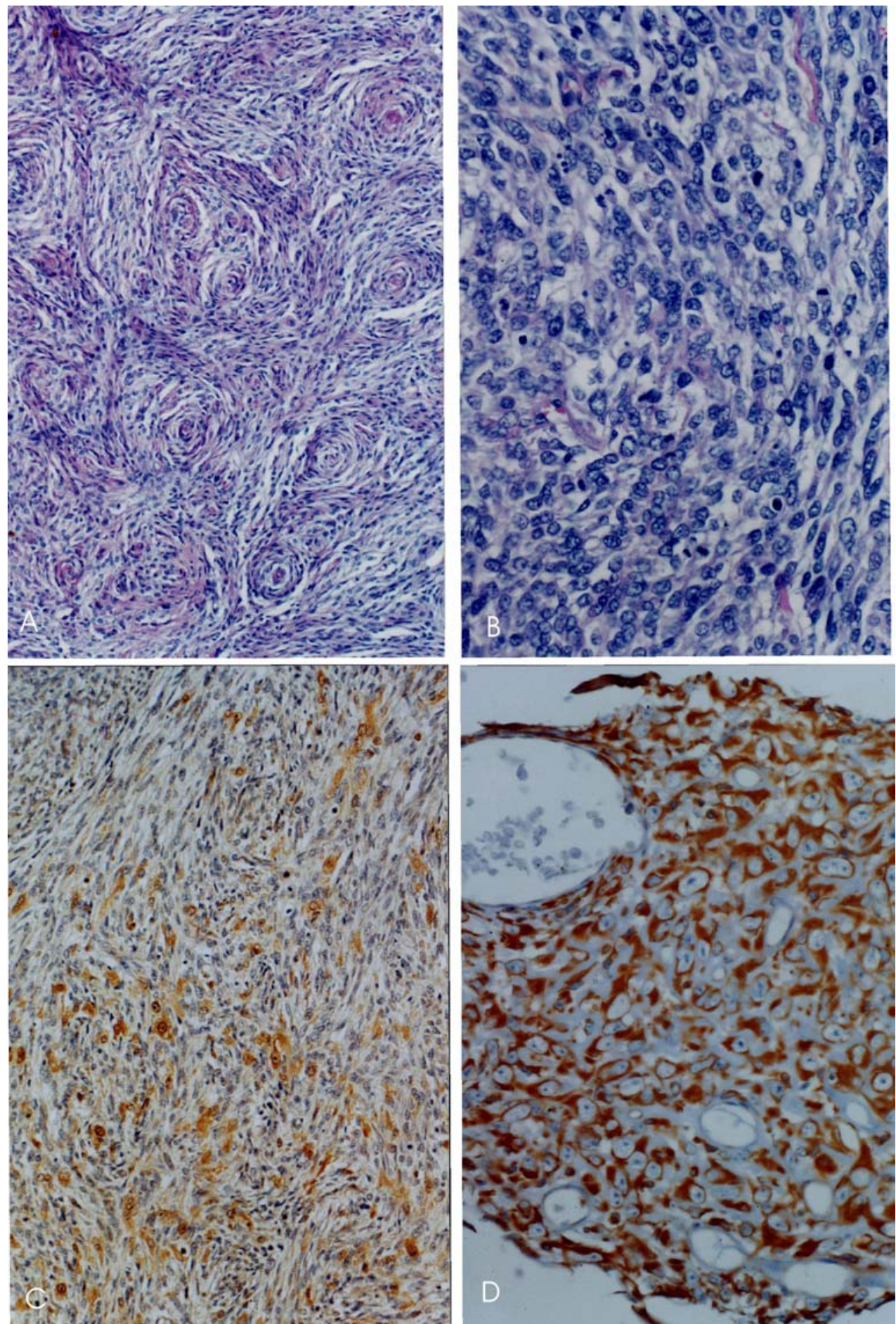

Figura 1. A e B: Caso 1 - Cão, tumor maligno da bainha de nervo periférico. A: Proliferação de células neoplásicas formando feixes, dispostas em paliçada ou ninhos. HE, 214,3X. B: Células neoplásicas pleomórficas, variando de fusiformes á poligonais, citoplasma basofílico granular, com bordas pouco definidas, núcleos grandes hipocromáticos, variando de oval a alongado, com nucléolo proeminente, por vezes múltiplos. Muitas figuras de mitose. HE, 428,6X. C: Caso 2- Expressão forte difusa da proteína S100 nas células neoplásicas. Estreptavidinabiotina-peroxidase, hematoxilina de Harris, 214,3X. D: Caso 3 - Expressão forte difusa de vimentina no citoplasma das células neoplásicas e vasos. Estreptavidina-biotina-peroxidase, hematoxilina de Harris, 428,6X. 


\section{REFERÊNCIAS BIBLIOGRÁFICAS}

BREHM, D.B.; VITE, C.H.; STEINBERG, H.S. et al. A retrospective evaluation of 51 cases of peripheral nerve sheath tumors in the dog. $J$. Am. Anim. Hosp. Assoc.,v.31, p.349-224,358 1995.

CHIJIWA, K.; UCHIDA, K.; TATEYAMA, S. Immunohistochemical evaluation of canine peripheral nerve sheath tumors and other soft tissue sarcomas. Vet. Pathol., v.41, p.307-318, 2004.

CORDY, D.R. Tumors of the nervous system. In: Moulton, J.E. Tumors in domestic animals. 3.ed. Berkeley: California, 1990. p.610-656.

GARCÍA, P.; SÁNCHEZ, B.; SÁNCHEZ, M.A.; GONZÁLEZ, M.; ROLLÁN, E.; FLORES, J.M. Epithelioid malignant peripheral nerve sheath tumor in a dog. J. Comp. Pathol., v.131, p.87-91, 2004.

JUBB, K.V.F.; KENNEDY, P.C.; PALMER, N. Pathology of domestic animals. 4.ed. San Diego: Academic, 1993. 3v.

KIM, D.Y.; CHO, D.Y.; KIM, D.Y. ET AL. Malignant peripheral nerve sheath tumor with divergent mesenchymal differentiations in a dog. J. Vet. Diagn. Invest., v.15, p.174-178, 2003.

KOESTNER, A.; BILZER, T.; FATZER, R. et al. Histological classification of tumors of the nervous system of domestic animals. 2.ed. Washington, 1999.

LECOUTEUR, R.A. Tumors of nervous system. In: WITHRAW, S.J. Small animal oncology. 2.ed. Philadelphia: Sauders, 1996. p.393-419.
PATNAIK, A.K.; ZACHOS, T.A.; SAMS, A.E. et al. Malignant nerve-sheath tumor with divergent and glandular differentiation in a dog: A case report. Vet. Pathol., v.39, p.406-410, 2002.

PLATT, S.R.; GRAHAM, J.; CHRISMAN C.L. et al. Magnetic resonance imaging and ultrasonography in the diagnosis of a malignant peripheral nerve sheath tumor in a dog. Vet Radiol. Ultrasound, v.40, p.367-371, 1999.

SAWAMOTO, O.; YAMATE, J.; KUWAMURA, M. et al. A canine peripheral nerve sheath tumor including peripheral nerve fibers. J. Vet. Med. Sci., v.61, p.1335-1338, 1999.

SIMPSON, D.J.; BECK, J.A.; ALLAN, G.S. et al. Diagnosis and excision of a brachial plexus nerve sheath tumour in a dog. Aust. Vet. J., v.77, p.222-224, 1999.

STOICA, G.; TASCA, S.I.; KIM,H.T. Point mutation of neu oncogene in animal peripheral nerve sheath tumors. Vet. Pathol., v.38, p.679$688,2001$.

SUMMERS, B.A.; CUMMINGS, J.F.; LAHUNTA, A. Neoplasia and the peripheral nervous system. In: Veterinary neuropathology. St. Louis, 1995. p.472-481.

VASILOPULOS, R.J.; MACKIN, A.J.; JENNINGS, D.; READ, R. What is your neurologic diagnosis? Nerve sheath tumor involving the brachial plexus with involvement of the spinal cord. J. Am. Vet. Med. Assoc., v.210, p.1397-1399, 2002. 